\title{
The parsimonious property of cut covering problems and its applications
}

\author{
Dimitris Bertsimas ${ }^{\mathrm{a}, *, 1}$, Chungpiaw Teo ${ }^{\mathrm{b}}$ \\ ${ }^{a}$ Sloan School of Management and Operations Research Center, MIT, Room E53-359, 50 Memorial Drive, \\ Cambridge, MA 02142-1347, USA \\ ${ }^{\mathrm{b}}$ Department of Decision Sciences, National University of Singapore, Singapore
}

Received 1 January 1996; revised 1 May 1997

\begin{abstract}
We consider the analysis of linear programming relaxations of a large class of combinatorial problems that can be formulated as problems of covering cuts, including the Steiner tree, the traveling salesman, the vehicle routing, the matching, the $T$-join and the survivable network design problem, to name a few. We prove that all of the problems in the class satisfy a nice structural property, the parsimonious property, generalizing earlier work by Goemans and Bertsimas (1993). We utilize the parsimonious property to establish worst-case bounds between the gap of the IP and LP values for the class of $0-1$ proper functions, leading to a new 2-approximation algorithm for this class of problems. We also extend the parsimonious property to a class of cut-covering problems that model certain instances of the edge-disjoint path problem. (C) 1997 Elsevier Science B.V.
\end{abstract}

Keywords: Integer programming; LP relaxations; Cutset formulations; Parsimonious property

\section{Introduction}

Given an undirected graph $G=(V, E)$ with nonnegative costs $c_{e}$ defined for every $e \in E$, we consider the class of problems described by the following integer programming formulation:

$\mathrm{IZ}_{f}(D)=\operatorname{minimize} \quad \sum_{e \in E} c_{e} x_{e}$

\footnotetext{
*Corresponding author. Tel.: +617-253-4223; e-mail: dbertsim@aris.mit.edu.

${ }^{1}$ Research partially supported by a Presidential Young Investigator Award DDM-9158118 with matching funds from Draper Laboratory.
}

$$
\begin{gathered}
\text { subject to } \sum_{e \in \delta(i)} x_{e}=f(i), \quad i \in D \subseteq V, \\
\sum_{e \in \delta(S)} x_{e} \geqslant f(S), \quad S \subset V, \\
x_{e} \in Z_{+}
\end{gathered}
$$

where $f: 2^{V} \rightarrow Z_{+}$is a given set function, and $\delta(S)=$ $\{e=(i, j) \in E \mid i \in S, j \in V \backslash S\}$. By selecting different set functions $f(S)$ and different sets $D$ we can model a large class of combinatorial problems, including the Steiner tree, the traveling salesman, the vehicle routing, the matching, $T$-join and survivable network design problems (see [6]).

Let $\operatorname{IP}_{f}(D)$ be the underlying feasible space of $\mathrm{IZ}_{f}(D)$. We denote the LP relaxation as $P_{f}(D)$, in 
which we replace constraints $x_{e} \in Z_{+}$with $x_{e} \geqslant 0$. We denote the value of the LP relaxation as $Z_{f}(D)$. Goemans and Bertsimas [4] studied the survivable network design problem, in which the objective is to design a network at minimum cost that satisfies connectivity requirements (for each pair $(i, j)$ of nodes in $V$, the solution should contain at least $r_{i j}$ edge disjoint paths) and considered an integer programming formulation of the type $\operatorname{IP}_{f}(D)$ with $f(S)=\max _{(i, j) \in \delta(S)} r_{i j}, D=\emptyset$. They showed the following property, which they call the parsimonious property, which in our notation can be stated as:

Theorem 1 (Goemans and Bertsimas [4]). If the costs $c_{e}$ satisfy the triangle inequality $\left(c_{i j} \leqslant c_{i k}+c_{k j}\right.$ for all $i, j, k \in V)$, then for the survivable network design problem $\left(f(S)=\max _{(i, j) \in \delta(S)} r_{i j}\right)$, for all $D \subseteq V$ :

$Z_{f}(D)=Z_{f}(\emptyset)$

In other words, the degree constraints are unnecessary for the LP relaxation in the survivable network design problem. They further examine several sometimes surprising structural and algorithmic properties of the LP relaxation, and examine the worst-case behavior of $\mathrm{IZ}_{f}(\emptyset) / Z_{f}(\emptyset)$ for the survivable network design problem. Goemans and Williamson [6], Williamson et al. [17] and Goemans et al. [5] show interesting worst-case bounds on the ratio $1 Z_{f}(\emptyset) / Z_{f}(\emptyset)$ for more general set functions $f(S)$.

Our goal in this paper is to understand the class of problems for which the parsimonious property holds and examine several implications of the parsimonious property. The contributions of this paper are as follows:

1. We continue the program started in [4] by identifying a set of conditions on the set function $f(S)$, for which the parsimonious property holds. In this way we prove that a large collection of classical combinatorial problems satisfy it including the matching problem, the $T$-join problem, a relaxation of the vehicle routing problem, some disjoint path problems, and some $b$-matching problems. In particular all problems considered in Goemans and Williamson [6] satisfy it. We also find that if the set function $f(S)$ does not satisfy this set of conditions, the parsimonious property does not hold. Goemans [3] has also independently developed this generalization using the technique in [4]. We prove the parsimonious property by extending an observation of Frank [2] for matching problems.

2. We use the parsimonious property to give simple proofs of the integrality of some polyhedra $P_{f}(D)$.

3. We further extend the parsimonious property under more general conditions and examine its implications to the disjoint path problem. We find that this extension is the source for several results in this area.

4. We offer a new proof technique that utilizes the parsimonious property to find bounds on the ratio $\mathrm{IZ}_{f}(\emptyset) / Z_{f}(\emptyset)$, when $f$ is $0-1$ proper (defined later). Our proof technique leads to a new approximation algorithm for this class of problems.

The paper is structured as follows. In Section 2, we introduce the properties of the set function $f(S)$ that imply the parsimonious property and examine classical combinatorial problems that can be modeled in this way. In Section 3, we prove the parsimonious property as well as the dual integral parsimonious property. In Section 4, we examine applications of the parsimonious property to the integrality of certain polyhedra $P_{f}(D)$. In Section 5 , we introduce a new proof technique to bound the ratio $\mathrm{IZ}_{f}(\emptyset) / Z_{f}(\emptyset)$. This proof technique gives rise to a new approximation algorithm for the problems considered in Goemans and Williamson [6]. In Section 6, we further extend the parsimonious property and apply it to the analysis of the disjoint path problem.

\section{Parsimonious set functions}

In their study of the approximability of problems in the class $\operatorname{IP}_{f}(\emptyset)$, Goemans and Williamson [6] (for the case that $f(S)$ takes values in $\{0,1\})$ and Williamson et al. in [17] (for the case that $f(S)$ takes values in $Z_{+}$) introduce the following set of conditions for the set function $f(S)$.

Conditions A (Proper set functions):

1. $f(\emptyset)=0$.

2. Symmetry: $f(S)=f(V \backslash S)$ for all $S \subseteq V$.

3. Properness: If $S \cap T=\emptyset, S, T \subset V$, then $f(S \cup T)$ $\leqslant \max \{f(S), f(T)\}$. 
We next introduce the following set of conditions:

\section{Conditions B (Parsimonious set functions):}

1. $f(\emptyset)=0$.

2. Symmetry: $f(S)=f(V \backslash S)$ for all $S \subseteq V$.

3. Node subadditivity $(N S)$ : If $S \cap\{x\}=\emptyset$, then $f(S \cup\{x\}) \leqslant f(S)+f(\{x\})$.

4. Quasi-supermodularity $(Q S)$ : For all $S, T \subseteq V$, $S \cap T \neq \emptyset$. Either

$$
f(S)+f(T) \leqslant f(S \cup T)+f(S \cap T)
$$

or

$$
f(S)+f(T) \leqslant f(S \backslash T)+f(T \backslash S) .
$$

We also introduce the general subadditivity condition:

Subadditivity: If $S \cap T=\emptyset$, then $f(S \cup T) \leqslant f(S)$ $+f(T)$.

The QS property was also introduced in the paper of Goemans et al. [5], who used the term weakly supermodular.

Conditions B are more general than Conditions A:

Proposition 1 (Goemans et al. [5]). Let $f$ be a symmetric, set function with $f(\emptyset)=0$. Then, if $f$ is proper, it is quasi-supermodular and node subadditive.

Proof. If $f$ is a proper function, then clearly $f$ is node subadditive. Among the terms $f(S \cap T), f(S \cup T)$, $f(S \backslash T), f(T \backslash S)$, say $f(S \cap T)$ attains the minimum. By properness, $f(S) \leqslant \max (f(S \cap T), f(S \backslash T))=$ $f(S \backslash T)$, and $f(T) \leqslant \max (f(S \cap T), f(T \backslash S))=$ $f(T \backslash S)$, and so $f(S)+f(T) \leqslant f(S \backslash T)+f(T \backslash S)$. The other cases follow similarly from symmetry of $f$.

\subsection{Examples of problems}

In Table 1 below we review several classical combinatorial problems formulated using the cut-set formulation $\mathrm{IP}_{f}(\emptyset)$ for $f$ satisfying Conditions $\mathrm{A}$, (and therefore B). All these problems are shown to be proper in [6].
We next describe problems that are parsimonious but not proper.

\subsubsection{The b-matching problem with $b(i) \in\{a, a+1\}$}

Given numbers $b(i)$ such that $\sum_{i \in V} b(i)=2 r$, the problem can be modeled in the form of $\operatorname{IP}_{f}(D)$ with $D=V$ and

$$
f(S)=\left\{\begin{array}{rr}
1 \quad \forall S:|S| \geqslant 2 \text { and } \\
& \sum_{i \in S} b(i)=2 k+1, \\
b(i) \quad & S=\{i\}, V \backslash\{i\} .
\end{array}\right.
$$

Notice that the function $f$ is not proper, because for $S, T$ disjoint whose union is $V \backslash\{i\}$ the definition is violated. However, the function $f(S)$ is QS. While $f$ is not subadditive for general sets $S, T$, it is node subadditive if $b(i) \in\{a, a+1\}$.

\subsubsection{The capacitated tree problem}

Given a graph $G=(V \cup\{0\}, E)$, nonnegative demands $d_{i}, i \in V$, a depot 0 , costs $c_{e}, e \in E$. We would like to design a tree of minimum cost such that each subtree from the depot has demand at most $Q$. The capacitated tree problem is a popular relaxation of the vehicle routing problem. A valid cutset formulation of the capacitated tree problem is of the type $\operatorname{IP}_{f}(\emptyset)$ with

$$
\begin{aligned}
& f(S)=\frac{\sum_{i \in S} d_{i}}{Q}, \\
& f(S \cup\{0\})=\frac{\sum_{i \in V \backslash S} d_{i}}{Q} .
\end{aligned}
$$

It is obvious that $f(S)$ does not satisfy Conditions A, since it is not proper but it satisfies Conditions B. It is clearly symmetric and subadditive as we show below, for $S, T \subset V$ such that $S \cap T=\emptyset$

$$
f(S \cup T)=\frac{\sum_{i \in S \cup T} d_{i}}{Q}=f(S)+f(T),
$$

$$
\begin{aligned}
f((S \cup\{0\}) \cup T) & =\frac{\sum_{i \in V \backslash(S \cup T)} d_{i}}{Q} \\
& \leqslant f(S \cup\{0\}) \\
& \leqslant f(S \cup\{0\})+f(T) .
\end{aligned}
$$


Table 1

Set functions $f(S)$ satisfying conditions $\mathrm{A}$ (and therefore $\mathrm{B}$ ) modeling various integer programming problems $\mathrm{IP}_{f}(\emptyset)$ satisfying Conditions $\mathrm{A}$

\begin{tabular}{lll}
\hline Problem & $f(S)$ & Conditions \\
\hline Spanning tree & 1, for all $S \neq \emptyset, V$ & $\mathrm{~A}$ \\
Steiner tree & 1, if $S \cap T \neq \emptyset, T$ & $\mathrm{~A}$ \\
Shortest path & 1, if $|S \cap\{s, t\}|=1$ & $\mathrm{~A}$ \\
Generalized Steiner tree & 1, if $S \cap T_{i} \neq \emptyset, T_{i}, i=1, \ldots, k$ & $\mathrm{~A}$ \\
Nonbipartite matching & 1, if $|S|=2 k+1$ & $\mathrm{~A}$ \\
$T$-join & 1, if $|S \cap T|=2 k+1$ & $\mathrm{~A}$ \\
Network survivability & $\max _{(i, j) \in \delta(S)} r_{i j}, r_{i j} \geqslant 0$ & $\mathrm{~A}$ \\
$k$-connected graph & $k$ for all $S \neq \emptyset, V$ & $\mathrm{~A}$ \\
Tree partitioning & 1, if $|S| \leqslant k,|S| \geqslant n-k$ & $\mathrm{~A}$ \\
Point-to-point connection & 1, if $|S \cap C| \neq|S \cap D|$ & $\mathrm{A}$ \\
\hline
\end{tabular}

It is also QS, since for $S, T \subset V$,

$$
\begin{aligned}
f(S \cup T)+f(S \cap T) & =\frac{\sum_{i \in S \cup T} d_{i}}{Q}+\frac{\sum_{i \in S \cap T} d_{i}}{Q} \\
& =\frac{\sum_{i \in S} d_{i}}{Q}+\frac{\sum_{i \in T} d_{i}}{Q} \\
& =f(S)+f(T),
\end{aligned}
$$

$$
\begin{aligned}
& f((S \cup\{0\}) \cup T)+f((S \cup\{0\}) \cap T) \\
& \quad=\frac{\sum_{i \in V \backslash(S \cup T)} d_{i}}{Q}+\frac{\sum_{i \in S \cap T} d_{i}}{Q} \\
& \quad \leqslant f((S \cup\{0\}) \backslash T)+f(T \backslash(S \cup\{0\})),
\end{aligned}
$$

$$
\begin{aligned}
& f((S \cup\{0\}) \cup(T \cup\{0\})) \\
&+f((S \cup\{0\}) \cap(T \cup\{0\})) \\
&= \frac{\sum_{i \in V \backslash(S \cup T)} d_{i}}{Q}+\frac{\sum_{i \in V \backslash(S \cap T)} d_{i}}{Q} \\
&= f(S \cup\{0\})+f(T \cup\{0\}) .
\end{aligned}
$$

\section{The parsimonious property}

The cut-set formulation introduced in the previous section captures many of the classical optimization problems studied in the literature. It is thus interesting and indeed surprising that the parsimonious property holds for the LP relaxations of these problems. In the remainder of this section, we prove the parsimonious property using linear programming duality extending an observation of Frank [2] for the matching problem to the general class of problems $P_{f}(\emptyset)$.

The dual of $P_{f}(D)$ is as follows:

$$
\begin{aligned}
\mathrm{DZ}_{f}(D)=\operatorname{maximize} & \sum_{S \subset V} y(S) f(S) \\
\text { subject to } & \sum_{S: e \in \delta(S)} y(S) \leqslant c(e), \quad e \in E, \\
& y(S) \geqslant 0, \quad S \subset V, S \neq\{i\}, \\
& \forall i \in D .
\end{aligned}
$$

Let $\mathrm{DP}_{f}(D)$ be the dual polyhedron and $\mathrm{DZ}_{f}(D)$ denote the optimal objective value. To prove the parsimonious property using a dual argument, we only need to show that among all dual optimal solutions to $\mathrm{DP}_{f}(D)$, we can always choose one with $y(v) \geqslant 0$ for all $v \in D$. This solution is then feasible to $\operatorname{DP}_{f}(\emptyset)$. Let $\mathscr{T}$ be a collection of sets (subsets of $V$ ). We call this family of sets laminar if for all $A, B \in \mathscr{T}$ either $A \cap B=\emptyset$, or $A \subset B$, or $B \subset A$ (see [13]).

Theorem 2 (Parsimonious property). If the cost function $c$ satisfies the triangle inequality, and $f$ is a parsimonious set function, then

$Z_{f}(D)=Z_{f}(\emptyset)$ for all $D \subseteq V$.

Proof. Let $y$ be a dual optimal solution in $\mathrm{DP}_{f}(D)$. By the QS property, we may assume that the set $\widetilde{F}:=\{S: y(S)>0\}$ is laminar, since we can always replace two intersecting sets $S$ and $T$ by $S \backslash T, T \backslash S$ or 
$S \cap T, S \cup T$. Suppose there exists a $v \in D$ such that $y(v)<0$. For all $A \in \mathscr{F}$ containing $v$, we replace $A$ by $V \backslash A$, i.e., we set

$$
y(A) \leftarrow 0, \quad y(V \backslash A) \leftarrow y(V \backslash A)+y(A) .
$$

In this way, we obtain another dual optimal solution with no member of $\mathscr{F}$ containing $v$. Note that $\mathscr{F}$ is still laminar.

Let $p(e)=\sum_{S: e \in \delta(S)} y(S)$. By dual feasibility, $p(e) \leqslant c(e)$. We may assume that there is a $u \in V$ such that $e=(u, v)$ and $p(e)=c(e)$, since we can increase $y(v)$ otherwise. Let $A$ be a maximal member of $\mathscr{F}$ containing $u$. Let $\Delta=\min (-y(v), y(A))$. We modify the dual solution as follows:

$y(v) \leftarrow y(v)+\Delta$,

$y(\overline{A \cup\{v\}}) \leftarrow y(\overline{A \cup\{v\}})+\Delta$,

$y(A) \leftarrow y(A)-\Delta$.

To check for feasibility of this modified solution, we only need to consider edges of the form $(v, w)$ where $w$ is not in $A$. Note that by the construction of $\mathscr{F}, p(u, w)=p(v, w)+p(u, v)-$ $2 y(v)$. Hence, $c(v, w) \geqslant c(u, w)-c(u, v)=c(u, w)-$ $p(u, v) \geqslant p(u, w)-p(u, v)=p(v, w)-2 y(v) \geqslant p(v, w)$ +24 . Thus, the modified solution is dual feasible. Since $f(S)$ is symmetric and node subadditive, the objective value of the new dual solution does not decrease. Therefore, the modified solution is also optimal. By repeating this procedure, we can construct a dual optimal solution with $y(v) \geqslant 0$ for all $v$ in $D$.

Notice that if $y$ in the above proof takes only integral values, then $\Delta$ can be chosen to be integral. This yields an integral analogue of the parsimonious property in a dual sense. Let $\operatorname{DIZ}_{f}(D)$ denote the optimal objective value over $\operatorname{DP}_{f}(D)$ with integrality constraints on $y(S)$.

Theorem 3 (Dual integral parsimonious property). If $f$ is parsimonious, and $c$ satisfies the triangle inequality, then $\mathrm{DIZ}_{f}(\emptyset)=\mathrm{DIZ}_{f}(D)$.

The parsimonious property does not hold if we relax either the QS or the node subadditivity property.

Consider the set function $f$ on 3 nodes as follows: $f\left(v_{1}\right)=f\left(\left\{v_{2}, v_{3}\right\}\right)=1, f(S)=0$, otherwise. Then clearly $f$ is QS, but it is not node subadditive. In this case the parsimonious property does not hold, as the polyhedron $P_{f}(V)$ is empty.

On the other hand, subadditivity alone does not guarantee the parsimonious property. Define $f$ on 4 nodes as follows: $f(S)=1$ if $|S|=1$ or $3, f(S)=2$, otherwise. Then $f$ is clearly subadditive and symmetric. In this instance, $P_{f}(V)$ is again empty, since if $x\left(v_{i}, v_{j}\right)>0$ and $x\left(\delta\left(v_{i}\right)\right)=x\left(\delta\left(v_{j}\right)\right)=1$, then $x\left(\delta\left(\left\{v_{i}, v_{j}\right\}\right)\right)<2$.

Although the condition that the cost function $c$ satisfies the triangle inequality seems restrictive, for problems of the form $\operatorname{IP}_{f}(\emptyset)$, i.e., with no degree constraints, we can ensure that this condition is met by the following transformation. Let $c^{\prime}(u, v)$ denote the shortest path between $u$ and $v$ with $c$ as the length function. Clearly, $c^{\prime}$ satisfies the triangle inequality. Let $\mathrm{IZ}_{f}^{\prime}(\emptyset)$ and $Z_{f}^{\prime}(\emptyset)$ denote the respective solution value with $c^{\prime}$ as the objective function. We use the technique introduced in [4] to prove:

\section{Theorem 4.}

$Z_{f}^{\prime}(\emptyset)=Z_{f}(\emptyset), \mathrm{IZ}_{f}^{\prime}(\emptyset)=\mathrm{IZ}_{f}(\emptyset)$.

Proof. Let $x$ be an optimal solution to $P_{f}^{\prime}(\emptyset)$ (or $\left.\operatorname{IP}_{f}^{\prime}(\emptyset)\right)$. Consider an edge $e=(u, v)$ such that $c^{\prime}(e)$ $<c(e)$. Let $P$ be a shortest path (with respect to $c$ ) linking $u$ and $v$. Then $P \neq\{e\}$, and $c^{\prime}(g)=c(g)$ for each edge $g$ on $P$. If $x(e)>0$, we let $x(e) \leftarrow 0$, and $x(g) \leftarrow x(g)+x(e)$ for each edge $g$ on $P$. Clearly the solution is still feasible, and since $c^{\prime}(e)=\sum_{g \in P} c(g)$, the cost of the new solution is the same, thus it is also optimal. Repeating this procedure, we have $x(e)>0$ only when $c^{\prime}(e)=c(e)$, thus proving the theorem.

\section{Applications in proofs of integrality of polyhedra}

An important direction of research in integer programming is the development of techniques to show integrality of the associated polyhedra for integer programming problems. Perhaps the most common proof technique is algorithmic. Researchers develop an optimal algorithm for a combinatorial optimization problem, which at the same time shows integrality of a proposed formulation for the problem. In this section we show that the parsimonious property leads to non- 
algorithmic, genuinely simple proofs of integrality of some polyhedra $P_{f}(D)$, yielding new simple proofs of some classical results as well as some new results.

A milestone in combinatorial optimization is the proof of integrality [1] of the perfect matching polyhedron. This result follows directly from the integrality of the $T$-join polyhedron, as the perfect matching polyhedron is a face of the $T$-join polyhedron. Surprisingly, we can derive the integrality of $T$-join polyhedron from that of the perfect matching polyhedron, using the parsimonious property. Let $\operatorname{Conv}(S)$ denote the convex hull of the set $S$.

Theorem 5. Let $f(S)=1$, if $|S \cap T|$ is odd, 0 otherwise. Then

$\operatorname{Conv}\left(\operatorname{IP}_{f}(\emptyset)\right)=P_{f}(\emptyset)$.

Proof. Let $f_{T}$ be the restriction of $f$ to $T$, defined on $S \subset T$. Note that $\operatorname{IP}_{f_{T}}(T)$ is just the perfect matching polyhedron on $T$. We show next that $\mathrm{IZ}_{f}(\emptyset)=Z_{f}(\emptyset)$ for all integral cost functions $c$. By Theorem 4 , we may assume $c$ satisfies the triangle inequality. The following inequalities are immediate:

$\mathrm{IZ}_{f}(\emptyset) \leqslant \mathrm{IZ}_{f_{\tau}}(\emptyset) \leqslant \mathrm{IZ}_{f_{r}}(T)$.

From the integrality of the perfect matching problem $\mathrm{I} Z_{f_{T}}(T)=Z_{f_{T}}(T)$. From the parsimonious property $Z_{f_{T}}(T)=Z_{f_{r}}(\emptyset) \leqslant Z_{f}(\emptyset)$, yielding that

$\mathrm{IZ}_{f}(\emptyset) \leqslant Z_{f}(\emptyset)$.

The reverse inequality holds trivially and so $\mathrm{IZ}_{f}(\emptyset)=Z_{f}(\emptyset)$, which shows integrality of the $T$-join polyhedron.

The shortest path polyhedron can be treated as a Steiner-1-connectivity polyhedron on two terminal nodes. Integrality of the polyhedron also follows easily from the parsimonious property. We generalize this result, using the parsimonious property, and show that the cut set formulation for the Steiner-2connected polyhedron with at most 5 terminal vertices is integral.

Theorem 6. For the Steiner-2-connected problem on at most 5 terminal nodes,

$\operatorname{Conv}\left(\operatorname{IP}_{f}(\emptyset)\right)=P_{f}(\emptyset)$.
Proof. It is well known [11] that the subtour elimination polyhedron for the traveling salesman problem, which can be represented as $P_{f}(V)$ with $f(S)=2$, on at most 5 nodes is integral. From the parsimonious property the result follows easily.

We next consider the uncapacitated multicommodity flow problem with a single source $s$, multiple sinks $D=\left\{t_{1}, t_{2}, \ldots, t_{k}\right\}$ modeled as $\operatorname{IP}_{f}(\emptyset)$, with $f(S)=|D \cap S|$ if $s \notin S$ and $f(S)=f(\bar{S})$ if $s \in S$. Algorithmically, the problem reduces to the computation of the corresponding shortest paths between the source and the sinks. Note that if $D=V$ the problem is the shortest path tree problem. We show next that the polyhedron $P_{f}(\emptyset)$ is integral. This result also follows from Johnson [7].

Theorem 7. For the uncapacitated multicommodity flow problem with a single source and multiple sinks,

$\operatorname{Conv}\left(\operatorname{IP}_{f}(\emptyset)\right)=P_{f}(\emptyset)$.

Proof. We can assume without loss of generality that the cost satisfies the triangle inequality. Clearly, $\mathrm{IZ}_{f}(\emptyset) \leqslant \mathrm{IZ}_{f}(V)$. Since $P_{f}(V)$ has only a single integral solution with $x\left(s, t_{i}\right)=1$ for each $i=1,2, \ldots, k$, $\mathrm{IZ}_{f}(V)=Z_{f}(V)$. By the parsimonious property, $Z_{f}(V)=Z_{f}(\emptyset)$. Thus, $\mathrm{IZ}_{f}(\emptyset) \leqslant Z_{f}(\emptyset)$, and the result follows.

\section{Applications in worst-case analysis}

In recent years there has been a lot of interest in the approximability of combinatorial optimization problems. Typically researchers propose a heuristic algorithm for an integer programming problem (a minimization problem) and compare the value of the heuristic to the value of the LP relaxation (or to the value of a dual feasible solution of the LP relaxation). A very nice and very general example of this approach is the $2(1-(1 /|T|))$ approximation algorithm $(T=\{v \in V: f(v)=1\})$ proposed in [4] for the problem $\operatorname{IP}_{f}(\emptyset)$ with $f$ being proper (Conditions $\mathrm{A}$ ) and taking values in $\{0,1\}$. A corollary of their result is the bound $\mathrm{IZ}_{f}(\emptyset) / Z_{f}(\emptyset) \leqslant 2(1-(1 /|T|))$. A distinct characteristic of their method is a reverse deletion step, in which edges that were added in the solution are deleted. 
In this section we propose a new proof method that shows that $\mathrm{IZ}_{f}(\emptyset) / Z_{f}(\emptyset) \leqslant 2(1-(1 /|T|))$ for $0-1$ proper functions.

\subsection{A proof technique to bound the ratio $\mathrm{IZ}_{f}(\emptyset) /$ $Z_{f}(\emptyset)$}

We consider problem $\operatorname{IP}_{f}(\emptyset)$ with $f$ being a $0-1$ proper function, and integral costs $c_{e}$. The latter is without loss of generality, since, if the costs are rational we can rescale them to integers by multiplying them by an appropriate large integer without affecting the optimal solution. Let $T=\{v \in V: f(v)=1\}$. Our proof technique uses the crucial observation that a minimal integral solution to the problem must be a forest, and thus has at most $|T|-1$ edges. An integral solution is minimal if it does not properly contain any other feasible integral solution.

Theorem 8 (Goemans and Williamson [6]). If $f$ is a 0-1 proper function

$\mathrm{IZ}_{f}(\emptyset) \leqslant 2\left(1-\frac{1}{|T|}\right) Z_{f}(\emptyset)$

Proof. For the purpose of contradiction we assume the contrary. Therefore, there exists a counterexample on the least number of nodes, with $f$ proper and $c$ nonnegative. We may further assume that $\sum_{e \in E} c(e)$ is minimal.

Suppose there is a $v$ with $f(v)=0$. Let $f^{\prime}$ denote the restriction of $f$ on $V \backslash\{v\}$. It can easily be checked that $f^{\prime}$ is still proper. By the minimality of the counterexample,

$\mathrm{IZ}_{f^{\prime}}(\emptyset) \leqslant 2\left(1-\frac{1}{|T|}\right) Z_{f^{\prime}}(\emptyset)$.

Since the optimal solution in $\mathrm{IP}_{f^{\prime}}(\emptyset)$ is also feasible in $\operatorname{IP}_{f}(\emptyset), \mathrm{IZ}_{f}(\emptyset) \leqslant \mathrm{IZ}_{f}(\emptyset)$. From Theorem 4 , by using the shortest path distances $Z_{f}(\emptyset)=Z_{f}^{\prime}(\emptyset)$ and $Z_{f^{\prime}}(\emptyset)=Z_{f^{\prime}}^{\prime}(\emptyset)$. But, $Z_{f^{\prime}}^{\prime}(\emptyset)=Z_{f}^{\prime}(\{v\})$. By the parsimonious property $Z_{f}^{\prime}(\emptyset)=Z_{f}^{\prime}(\{v\})=Z_{f^{\prime}}^{\prime}(\emptyset)$. Therefore,

$\mathrm{IZ}_{f}(\emptyset) \leqslant 2\left(1-\frac{1}{|T|}\right) Z_{f}(\emptyset)$

which is a contradiction. So we may assume $f(v)=1$ for all $v$.
If there is an edge $e=(u, v) \in E$ with $c_{e}=0$, then by contracting this edge, and treating $\{u, v\}$ as a supernode, we restrict the problem to one of strictly smaller size. By the minimality of the counterexample, there exists a solution that satisfies the theorem. By introducing the edge $(u, v)$, with no extra cost since $c_{e}=0$, if necessary, we obtain a solution feasible to the original problem and the theorem holds. Therefore, we may assume $c_{e}>0$ for all $e$.

Now let $y(v)=\frac{1}{2}$ for all $v$ and consider the cost function $c^{\prime}$ where $c_{e}^{\prime}=c_{e}-1\left(c_{e}^{\prime} \geqslant 0\right.$ from the previous paragraph). By the minimality of $c$, there exist $x, y^{\prime}$ such that $x \in \operatorname{IP}_{f}(\emptyset), \sum_{S: e \in \delta(S)} y^{\prime}(S) \leqslant c_{e}^{\prime}$ and

$\sum_{e} c_{e}^{\prime} x_{e} \leqslant 2\left(1-\frac{1}{|T|}\right) \sum_{S} y^{\prime}(S) f(S)$.

Since $f$ is $0-1, x_{e}$ corresponds to a forest and, therefore,

$$
\begin{aligned}
\sum_{e} x_{e} \leqslant & |T|-1=2\left(1-\frac{1}{|T|}\right) \\
& \times \sum_{S} y(v)=2\left(1-\frac{1}{|T|}\right) \sum_{S} f(v) y(v) .
\end{aligned}
$$

The last equality holds, since we have shown that we can assume $f(v)=1$. Let $y^{*}=y^{\prime}+y$. Note that

$$
\sum_{S: e \in \delta(S)} y^{*}(S)=\sum_{S: e \in \delta(S)} y(S)+\frac{1}{2}+\frac{1}{2} \leqslant c_{e}^{\prime}+1=c_{e}
$$

and so $y^{*}$ is dual feasible. Therefore,

$$
\begin{aligned}
\sum_{e} c_{e} x_{e} & =\sum_{e} c_{e}^{\prime} x_{e}+\sum_{e} x_{e} \\
& \leqslant 2\left(1-\frac{1}{|T|}\right) \sum y^{*}(S) f(S)
\end{aligned}
$$

This is again a contradiction and the theorem follows.

Although the proof method in Theorem 8 is nonalgorithmic, it also leads to an algorithmic method to construct an approximate solution. It differs from the Goemans and Williamson's algorithm in that it needs a preprocessing step to compute pairwise shortest paths. With this in hand, we can discard all vertices with $f(v)=0$. We call these vertices the Steiner vertices. 
This approach avoids the critical reverse deletion step in the Goemans and Williamson's algorithm, at the expense of computing pairwise shortest paths. Our algorithm is as follows:

Approximation algorithm for $0-1$ proper functions

1. Compute the pairwise shortest path distances for all pairs of non-Steiner nodes.

2. Discard the set of Steiner nodes. Select an edge with the least cost. Merge the two end nodes into a supernode, delete all edges joining these two nodes.

3. Repeat Step 2 until two supernodes merge to form a set $S$ with $f(S)=0$. Let $e^{\prime}$ be the last edge selected. If there are no more non-Steiner nodes, go to Step 4. Else, for all edges remaining, reduce the cost coefficients by $c\left(e^{\prime}\right)$ and return to Step $1 .^{1}$

4. Replace the edges selected in Steps 2 and 3 by its corresponding shortest path. Delete edges (if necessary) if cycles exist in the subgraph selected.

For the Steiner tree problem, our algorithm emulates the MST heuristic on non-Steiner nodes with the pairwise shortest distance metric. In this respect, Theorem 8 generalizes the well-known fact that the MST heuristic gives a $2(1-(1 /|T|))$ approximate solution to the minimum Steiner tree problem.

For arbitrary proper functions $f$, as Goemans and Williamson [6] observe, we can construct a feasible solution by utilizing Theorem 8 . Let $p_{1}<p_{2}<\cdots<p_{n}$ be the distinct values of $f$, and for each $i, f_{p_{i}}(S)=1$ if $f(S) \geqslant p_{i}$ and 0 otherwise. Note that $f_{p_{i}}$ is proper $0-1$. By appending $p_{i}-p_{i-1}$ $\left(p_{0}=0\right)$ copies of the approximate solution to $f_{p_{i}}$ for each $i=1,2, \ldots, n$, we obtain a feasible solution which is within $2 \mathscr{H}\left(p_{1}, p_{2}, \ldots, p_{n}\right)$ times of the optimal solution, where $\mathscr{H}\left(p_{1}, p_{2}, \ldots, p_{n}\right)=\sum_{i=1}^{n}\left(p_{i}-\right.$ $\left.p_{i-1}\right) / p_{i}$. Similarly, for arbitrary QS functions we can use the results of [5] to find

$\mathrm{IZ}_{f}(\emptyset) \leqslant 2 \mathscr{H}\left(p_{1}, p_{2}, \ldots, p_{n}\right) Z_{f}(\emptyset)$.

\footnotetext{
${ }^{1}$ Note that the new cost coefficients obtained this way need not satisfy the triangle inequality. We need to perform another shortest path computation with these new cost coefficients.
}

\section{Weakly parsimonious functions and the disjoint path problem}

A natural question is whether node subadditivity and quasi-supermodularity (QS) are the most general conditions on the set function $f$ for the parsimonious property to hold. To this end, we introduce a third set of conditions on the set function $f$ :

Conditions $\mathbf{C}$ (Weakly parsimonious set functions):

1. $f(\emptyset)=0$.

2. Symmetry: $f(S)=f(V \backslash S)$ for all $S \subseteq V$.

3. Weak subadditivity (WS): If $S \cap\{x\}=\emptyset$, then $f(S \cup\{x\}) \leqslant f(S)$. We will then say that $x$ is a weakly Steiner vertex.

4. 2-Quasi-supermodularity (2-QS): For every three mutually crossing sets (two sets $S, T$ are crossing if all of $S \backslash T, T \backslash S, S \cap T$ are nonempty) at least two of them satisfy the QS property.

Compared with Conditions B (parsimonious set functions), weak subadditivity is stronger than node subadditivity, while the 2-QS property is a relaxation of the QS property. In other words, there are set functions $f$ satisfying one of conditions $B$ or $C$ but not the other.

In this section we show that the parsimonious property still holds for weakly parsimonious set functions and observe that these relaxed conditions provide a unifying understanding of several results on the disjoint path problem.

We next prove that the parsimonious property holds for weakly parsimonious functions, using the splitting technique of [9] and used in [4].

Definition 1 (Splitting operation). For some $u, w$ with $x(v, u)>0, x(v, w)>0$, we split $v$ using $u, w$ by the following modification:

- $x(v, u) \leftarrow x(v, u)-\varepsilon$,

- $x(v, w) \leftarrow x(v, w)-\varepsilon$,

- $x(u, w) \leftarrow x(u, w)+\varepsilon$.

Note that the splitting operation does not increase the objective value, if the cost function satisfies the triangle inequality.

Theorem 9. Let $D$ be the set of weakly Steiner vertices. If $c$ satisfies the triangle inequality, and $f$ is weakly parsimonious, then

$Z_{f}(D)=Z_{f}(\emptyset)$. 
Proof. Let $v \in D, u \in V$, and suppose $x(\delta(v))>$ $f(v), x(v, u)>0$, where $x$ is an optimal solution in $P_{f}(\emptyset)$. Consider the minimal tight sets that contains $v$ but not $u$. By the 2-QS property, there exist at most 2 such minimal sets, say $S_{1}$ and $S_{2}$. Then all tight sets containing $v$ but not $u$ must contain one of these two sets.

We show next that there is a $w$ in $S_{1} \cap S_{2}$ with $x(v, w)>0$. Assuming the contrary, then

$$
\begin{aligned}
f\left(S_{i}\right)= & x\left(\delta\left(S_{i}\right)\right)=x\left(\delta\left(S_{i} \backslash\{v\}\right)\right) \\
& +x\left(\delta\left(\{v\}, \overline{S_{i}}\right)\right)-x\left(\delta\left(\{v\}, S_{i}\right)\right) \\
\geqslant & f\left(S_{i} \backslash\{v\}\right)+x\left(\delta\left(\{v\}, \overline{S_{i}}\right)\right)-x\left(\delta\left(\{v\}, S_{i}\right)\right) .
\end{aligned}
$$

From weak subadditivity, $f\left(S_{i} \backslash\{v\}\right) \geqslant f\left(S_{i}\right)$, hence,

$x\left(\delta\left(\{v\}, \overline{S_{i}}\right)\right) \leqslant x\left(\delta\left(\{v\}, S_{i}\right)\right)$.

Since we have assumed that $x\left(\delta\left(\{v\}, S_{1} \cap S_{2}\right)\right)=0$, we rewrite the inequality for $i=1,2$ and obtain

$$
\begin{aligned}
& x\left(\delta\left(\{v\}, S_{2} \backslash S_{1}\right)\right)+x\left(\delta\left(\{v\}, \overline{S_{1} \cup S_{2}}\right)\right) \\
& \quad \leqslant x\left(\delta\left(\{v\}, S_{1} \backslash S_{2}\right)\right)
\end{aligned}
$$

and

$$
\begin{aligned}
& x\left(\delta\left(\{v\}, S_{1} \backslash S_{2}\right)\right)+x\left(\delta\left(\{v\}, \overline{S_{1} \cup S_{2}}\right)\right) \\
& \quad \leqslant x\left(\delta\left(\{v\}, S_{2} \backslash S_{1}\right)\right) .
\end{aligned}
$$

Hence $x\left(\delta\left(\{v\}, \overline{S_{1} \cup S_{2}}\right)\right) \leqslant 0$, which is a contradiction since $x(v, u)>0$ and $u \in \overline{S_{1} \cup S_{2}}$. Therefore, there exists a $w$ in $S_{1} \cap S_{2}$ with $x(v, w)>0$. By splitting at $v$ using $u, w$, we obtain another feasible optimal (because of the triangle inequality) solution. By repeating this procedure, we obtain an optimal solution in $P_{f}(D)$, thus proving the theorem.

The above proof actually yields the following:

Corollary 1. Let $G$ be an Eulerian multigraph and $x_{G}$ be the incidence vector of $G$. Let $f$ be an even, 2-QS set function. If $x_{G}$ is a feasible integral solution to $\operatorname{IP}_{f}(\emptyset)$ and $x(\delta(v))>f(\{v\})$ for some weakly Steiner vertex $v \in V$, then there exists $\{u, w\}$ and an edge splitting operation of $v$ at $\{u, w\}$, yielding a new Eulerian graph $G^{\prime}$ and a corresponding incidence vector $x_{G^{\prime}}$ that is a feasible integral solution to $\operatorname{IP}_{f}(\emptyset)$.
As we show next, this corollary provides a unifying way to understand several seemingly unrelated results for the edge-disjoint-path (EDP) problem.

\subsection{2-QS functions and the disjoint path problem}

Given an undirected graph $G=(V, E)$, a collection of source-sink pairs $\left\{s_{1}, t_{1}\right\}, \ldots,\left\{s_{k}, t_{k}\right\}$, the EDP problem asks whether there exists a collection of edge disjoint paths in $G$, each joining a source to its corresponding sink. Let $H$ denote the demand graph, with edge set $\left\{\left(s_{1}, t_{1}\right), \ldots,\left(s_{k}, t_{k}\right)\right\}$. Let $x_{G}(e)=1$ if $e \in G$ and let $x_{H}(e)=1$ if $e \in H$.

Clearly, a necessary condition for the existence of these paths is the cut-criterion:

$x_{G}(\delta(S)) \geqslant x_{H}(\delta(S)) \quad$ for all $S \subset V$.

There has been an extensive literature (see, for example, $[2,14]$ ) that finds conditions on $G$ and $H$, so that the cut-criterion is both necessary and sufficient for the existence of a solution to the EDP problem. Let $K_{n}, C_{n}$ denote, respectively, the complete graph and the cycle on $n$ nodes. We also denote the disjoint union of $m$ copies of $K_{n}$ by $m K_{n}$. The following results are known:

Theorem 10. If $G+H$ is Eulerian, and $H$ is either a double star or $a K_{4}$ or a $C_{5}$, then the cut-criterion is necessary and sufficient for the solvability of the EDP problem.

The case in which $H$ is a $2 K_{2}$ was proved by Rothschild and Whinston [12]. The double star case follows easily from their result. The $K_{4}$ case was proved by Seymour [15] and Lomonosov [8] independently. The $C_{5}$ case is due to Lomonosov [8]. See $[2,14]$ for nice proofs and exposition of these results.

At first sight these results appear to be unrelated without a unifying characteristic. We could use the theory developed in this section to identify the unifying characteristic of all the above results contained in Theorem 10. The central reason is that the set function $x_{H}(\delta(S))$ in these cases has the 2-QS property. In particular it is easy to prove the following proposition:

Proposition 2. The set function $x_{H}(\delta(S))$ has the 2$Q S$ property if and only if $H$ does not contain a $3 K_{2}$ or disjoint copies of $K_{3}$ and $K_{2}$. This in turn holds if and only if $H$ is a double star or a $K_{4}$ or a $C_{5}$. 
In order to see how Proposition 2 can be used to prove Theorem 10, let us rewrite the cut condition as follows:

$x_{G+H}(\delta(S)) \geqslant f(S)=2 x_{H}(\delta(S))$.

Under the assumptions of Theorem 10 and using Proposition 2, $x_{G+H}$ corresponds to a Eulerian graph (by assumption), while $f$ is an even, 2-QS set function. Let $D=V \backslash\left\{s_{1}, t_{1}, \ldots, s_{k}, t_{k}\right\}$ be the nodes in $G$ that do not belong to a source-sink pair. In our context $D$ is the set of weakly Steiner vertices. Applying Corollary 1 we can then perform edge-splitting operations on the edges of $G$ to obtain a new graph $G^{\prime}$ that satisfies the cut criterion, but with edges incident only to the sources or sinks. The rest of the proof involves showing that $G^{\prime}$ has the set of edge-disjoint-paths joining each source-sink pair. This follows from a tedious case by case analysis which we omit here, as it is unrelated to the theme of the paper. By reversing the edge-splitting operations, we obtain a set of edgedisjoint-paths in $G$ that meets the cut criterion, thus proving Theorem 10 .

\section{Acknowledgements}

We would like to thank Michel Goemans for pointing out his unpublished work in [3] to us.

\section{References}

[1] J. Edmonds, Maximum matching and a polyhedron with $(0,1)$-vertices, J. Res. Nat. Bur. Standards 69B (1965) 125-130.

[2] A. Frank, Packing paths, circuits and cuts - a survey, in: B. Korte et al. (Eds.), Paths, Flows and VLSI-Layout, 1990, pp. 47-100.
[3] M.X. Goemans, A note on the parsimonious property, unpublished manuscript.

[4] M.X. Goemans, D. Bertsimas, Survivable networks, linear programming relaxations and the parsimonious property, Math. Programming 60 (1993) 145-166.

[5] M.X. Goemans, A.V. Goldberg, S. Plotkin, D.B. Shmoys, E. Tardos, D.P. Williamson, Improved approximation algorithms for network design problems, Proc. 5th ACMSIAM Symp. of Discrete Algorithms, 1993.

[6] M.X. Goemans, D.P. Williamson, A general approximation technique for constrained forest problems, Proc. 3rd ACMSIAM Symp. on Discrete Algorithms, 1992, pp. 307-316.

[7] E.L. Johnson, On cut-set integer polyhedra, Cahiers Centre Ehudes Rech. Oper. 17 (1975) 235-251.

[8] M.V. Lomonosov, Multiflow feasibility depending on cuts, Graph Theory Newsletter 9 (1979) 4.

[9] L. Lovász, On some connectivity properties of Eulerian graphs, Acta Math. Acad. Sci. Hungar. 28 (1976) 129-138.

[10] L. Lovász, M.D. Plummer, Matching Theory, North-Holland, Amsterdam, 1986.

[11] R.Z. Norman, On the convex polyhedra of the symmetric travelling salesman problem, Bull. Amer. Math. Soc. 61 (1955) 559.

[12] B. Rothschild, A. Whinston, Feasibility of two-commodity network flows, Oper. Res. 14 (1966) 1121-1129.

[13] A. Schrijver, Theory of Integer and Linear Programming, Wiley, Chichester, 1986.

[14] A. Schrijver, Short proofs on multicommodity flows and cuts, J. Combin. Theory Ser. B 53 (1991) 32-39.

[15] P.D. Seymour, Four-terminal flows, Networks 10 (1980) 79-86.

[16] D. Shmoys, D.P. Williamson, Analyzing the Held-Karp TSP bound: a monotonicity property with application, Inform. Process. Lett. 35 (1990) 281-285.

[17] D.P. Williamson, M.X. Goemans, M. Mihail, V.V. Vazirani, A primal-dual approximation algorithm for generalized Steiner network problems, Proc. 25th Ann. ACM Symp. on Theory of Computing, 1993, pp. 708-717.

[18] L.A. Wolsey, Heuristic analysis, linear programming and Branch and bound, Math. Prog. Study 13 (1980) 121-134. 